\title{
Longitudinal Progression of Cognitive Decline Correlates with Changes in the Spatial Pattern of Brain ${ }^{18}$ F-FDG PET
}

\author{
Sepideh Shokouhi ${ }^{1}$, Daniel Claassen ${ }^{2}$, Hakmook Kang ${ }^{3}$, Zhaohua Ding ${ }^{1}$, Baxter Rogers ${ }^{1}$, Arabinda Mishra ${ }^{1}$, and \\ William R. Riddle ${ }^{1}$, for The Alzheimer's Disease Neuroimaging Initiative \\ ${ }^{I}$ Department of Radiology and Radiological Sciences, Vanderbilt University Institute of Imaging Science, Nashville, Tennessee; \\ ${ }^{2}$ Department of Neurology, Vanderbilt University, Nashville, Tennessee; and ${ }^{3}$ Department of Biostatistics, Vanderbilt University, , \\ Nashville, Tennessee
}

Evaluating the symptomatic progression of mild cognitive impairment $(\mathrm{MCl})$ caused by Alzheimer disease $(\mathrm{AD})$ is practically accomplished by tracking performance on cognitive tasks, such as the Alzheimer Disease Assessment Scale's cognitive subscale (ADAS_ cog), the Mini-Mental Status Examination (MMSE), and the Functional Activities Questionnaire (FAQ). The longitudinal relationships between cognitive decline and metabolic function as assessed using ${ }^{18} \mathrm{~F}$-FDG PET are needed to address both the cognitive and the biologic progression of disease state in individual subjects. We conducted an exploratory investigation to evaluate longitudinal changes in brain glucose metabolism of individual subjects and their relationship to the subject's changes of cognitive status. Methods: We describe a method to determine correlations in ${ }^{18} \mathrm{~F}-\mathrm{FDG}$ spatial distribution over time. This parameter is termed the regional ${ }^{18} \mathrm{~F}-\mathrm{FDG}$ time correlation coefficient ( $\mathrm{rFTC}$ ). By using linear mixed-effects models, we determined the difference in the rFTC decline rate between controls and subjects at high risk of developing $A D$, such as individuals with $\mathrm{MCl}$ or the presence of apolipoprotein $\mathrm{E}$ (APOE)- $\varepsilon 4$ allele. The association between each subject's rFTC and performance on cognitive tests (ADAS_cog, MMSE, and FAQ) was determined with 2 different correlation methods. All subject data were downloaded from the Alzheimer Disease Neuroimaging Initiative. Results: The rFTC values of controls remained fairly constant over time $(-0.003$ annual change; 95\% confidence interval, -0.010 0.004). In MCl patients, the rFTC declined faster than in controls by an additional annual change of -0.02 ( $95 \%$ confidence interval, -0.030 to -0.010 ). In $\mathrm{MCl}$ patients, the decline in rFTC was associated with cognitive decline (ADAS_cog, $P=0.011$; FAQ, $P=$ 0.0016 ; MMSE, $P=0.004$ ). After a linear effect of time was accounted for, visit-to-visit changes in rFTC correlated with visitto-visit changes in all 3 cognitive tests. Conclusion: Longitudinal changes in rFTC detect subtle metabolic changes in individuals associated with variations in their cognition. This analytic tool may be useful for a patient-based monitoring of cognitive decline.

Key Words: Alzheimer imaging; ${ }^{18} \mathrm{~F}-\mathrm{FDG}$ PET; ${ }^{18} \mathrm{~F}-\mathrm{FDG}$ time correlation; mild cognitive impairment; longitudinal ${ }^{18} \mathrm{~F}-\mathrm{FDG}$ imaging

J Nucl Med 2013; 54:1564-1569

DOI: 10.2967/jnumed.112.116137

\footnotetext{
Received Oct. 22, 2012; revision accepted Apr. 4, 2013.

For correspondence contact: Sepideh Shokouhi, 1161 21st Ave. S., Medical Center North, AA-1105, Nashville, TN 37232-2310.

E-mail: sepideh.shokouhi@vanderbilt.edu

Published online Jul. 17, 2013.

COPYRIGHT $\odot 2013$ by the Society of Nuclear Medicine and Molecular Imaging, Inc.
}

$\mathbf{M}$ ild cognitive impairment (MCI) is a clinical entity defined as a transitional state from normal aging to dementia (1-3). Repeated standardized cognitive assessments allow for a clinical determination of progression to a dementia complex. Some of the most commonly used screening methods include the Alzheimer Disease Assessment Scale-cognitive subscale (ADAS_cog), the Mini Mental Status Examination (MMSE), and the Functional Activities Questionnaire (FAQ). However, there can arise situations for which these assessments fail to adequately capture the disease progression (4). Test-retest reliability, learning effects, floor or ceiling effects, and variability in disease progression $(5,6)$ can confound interpretation of these measures.

Quantitative biomarkers predictive of cognitive decline are needed to provide a precise measure of the rate of cognitive decline and potential response to therapy in subjects with mild MCI. ${ }^{18} \mathrm{~F}-\mathrm{FDG}$ PET measures cerebral metabolic rates of glucose utilization, which is representative of synaptic density and neuronal function (7). In MCI and Alzheimer disease (AD), distinct patterns of hypometabolism were observed in the posterior cingulate cortex, parietotemporal regions, and frontal lobes $(8-10)$, making ${ }^{18} \mathrm{~F}-$ FDG PET a well-established imaging biomarker of clinical AD $(11,12)$. Longitudinal ${ }^{18} \mathrm{~F}$-FDG PET assessments have been used to monitor metabolic decline (13-17) and determine how this decline associates with neuropsychologic patterns and concurrent changes in cognitive test scores $(18,19)$. Although these studies reveal metabolic and cognitive decline patterns in $\mathrm{AD}, \mathrm{MCI}$, and normal subject groups, they do not provide a direct comparison between temporal changes of cognition and metabolism in individual subjects. There still exists a need for patient-based methods to evaluate progression of cognitive and metabolic changes over time.

In this study, we took a different approach to assess ${ }^{18} \mathrm{~F}-\mathrm{FDG}$ PET changes. Instead of measuring the ${ }^{18}$ F-FDG uptake, we evaluated the similarity between follow-up ${ }^{18}$ F-FDG PET images and baseline images of each subject over time. Conventional ${ }^{18} \mathrm{~F}-$ FDG-based methods $(19,20)$ evaluate metabolism in brain regions that are typically affected in $\mathrm{AD}$, whereas we chose a multiregional approach by including areas that are known to be affected by AD and regions that are less or nonaffected. Our study allows a direct and straightforward comparison between metabolic changes $\left({ }^{18} \mathrm{~F}-\right.$ FDG) and cognitive changes (ADAS_cog, FAQ, and MMSE) in the same subject. Such patient-based comparison is potentially less sensitive to metabolic heterogeneities across subjects and might reveal interesting links between biologic and cognitive markers of the AD state. 


\section{MATERIALS AND METHODS}

Alzheimer Disease Neuroimaging Initiative (ADNI)

The data we present here is from the ADNI database. The ADNI was launched in 2003 by the National Institute on Aging, the National Institute of Biomedical Imaging and Bioengineering, the Food and Drug Administration, private pharmaceutical companies, and nonprofit organizations as a $\$ 60$ million, 5-y public-private partnership. The primary goal of ADNI has been to test whether serial MR imaging, PET, other biologic markers, and clinical and neuropsychologic assessment can be combined to measure the progression of $\mathrm{MCI}$ and early AD. Determination of sensitive and specific markers of very early AD progression is intended to aid researchers and clinicians to develop new treatments and monitor their effectiveness as well as lessen the time and cost of clinical trials. The Principal Investigator of this initiative is Michael W. Weiner, MD. ADNI is the result of efforts of many coinvestigators from a broad range of academic institutions and private corporations, and subjects have been recruited from over 50 sites across the United States and Canada.

\section{Subjects}

In this study, we included 51 subjects (27 MCI subjects and 24 controls), a random sample from ADNI subjects who had undergone at least 4 serial ${ }^{18}$ F-FDG PET scans using the same scanner, acquisition protocol, and image reconstruction method for the baseline and the follow-up scans. Furthermore, all subjects had T1-weighted MR image volumes and longitudinal scores of 3 different cognitive tests (MMSE, FAQ, and ADAS_cog). The average time difference between the first and last scan was $4.30 \pm 1 \mathrm{y}$ for both the MCI subjects and controls. All subjects gave written, informed consent before participation through their local Institutional Review Board. The key eligibility criteria of ADNI subject recruitment are available on the ADNI website (http://www.adni-info.org). Briefly, enrolled ADNI subjects were between the ages of 55 and $90 \mathrm{y}$, had a study partner able to provide an independent evaluation of functioning, and spoke either English or Spanish. All subjects had to be willing and able to perform all test procedures and imaging. We defined normal subjects as participants who were not taking cognitive-enhancing medications, such as acetylcholinesterase; were not treated for depression, thus not taking serotonin reuptake inhibitors or medications in that class because serotonin modulation can influence cerebral glucose metabolism (21); and did not convert to MCI or dementia over the course of the ADNI data collection period. With these exclusion data, we were able to ensure that our control group represented a homogeneous aging population and was without medication effects that could influence glucose metabolism. MCI subjects had memory complaints and objective memory loss measured by education-adjusted scores on the Wechsler Memory Scale Logical Memory II, had an absence of significant levels of impairment in other cognitive domains, and were able to preserve daily activities. Table 1 illustrates the demographic and clinical data of the subjects. At baseline, MCI patients evidenced greater impairment on ADAS_cog, FAQ, and MMSE than controls. A higher fraction of subjects in the MCI group (12 subjects with a single allele and 5 with a double allele) than in the normal group ( 5 subjects with a single allele and 1 with a double allele) had the presence of apolipoprotein $\mathrm{E}$ (APOE)- $\varepsilon 4$ allele.

\section{Clinical Ratings}

Longitudinally acquired clinical ratings were used to track the progression of cognitive decline. They included the ADAS_cog, FAQ, and MMSE. The ADAS_cog has a total score range from 0 of 70 points to measure the severity of cognitive impairment and is widely used in AD clinical trials (22). The MMSE test ranges from 0 to 30 and assesses orientation, immediate and delayed recall, attention, calculation, and language (23). The FAQ (scores, 0-30) is an assessment
TABLE 1

Clinical Data of ADNI Subjects Used for This Study

\begin{tabular}{lcc}
\hline \multicolumn{1}{c}{ Characteristic } & $\mathrm{MCl}$ & Normal \\
\hline No. of subjects & 27 & 24 \\
Female & 13 & 11 \\
Male & 14 & 13 \\
Average age at baseline & $74.6 \pm 7.2$ & $74.53 \pm 5.7$ \\
APOE- $\varepsilon 4$ & 17 & 6 \\
Baseline ADAS_cog & $11 \pm 4.7$ & $6 \pm 2.6$ \\
Baseline FAQ & $3.2 \pm 2.8$ & $0.1 \pm 0.6$ \\
Baseline MMSE & $27.4 \pm 1.4$ & $29.2 \pm 0.9$ \\
\hline
\end{tabular}

of an individual's ability to participate in functionally relevant activities, such as independent accomplishment of daily tasks (24). In ADAS_cog and FAQ, positive score changes indicate greater impairment, whereas in MMSE tests, a greater impairment is represented by a negative change. We chose these 3 tests because they are commonly used in the ADNI database and most other AD clinical trials. Furthermore, their relatively large scales of scores allow a better detection of subtle correlations between these tests and metabolic changes measured by ${ }^{18} \mathrm{~F}-\mathrm{FDG}$ PET.

\section{${ }^{18}$ F-FDG PET Data Acquisition}

All participating sites acquired the ${ }^{18}$ F-FDG PET imaging according to standardized ADNI protocols (www.Ioni.ucla.edu/ADNI/Data/). The serial PET images for our study were acquired at 17 different centers, and the data were obtained following the dynamic protocol of and preprocessed by ADNI. Dynamic scans were 30-min 6-frame scans acquired 30-60 min after injection. All frames were coregistered to the first frame, summed, and averaged to standard voxel size and resolution to create a single image volume.

\section{${ }^{18}$ F-FDG PET Regions of Interest (ROI)}

The gray matter fraction of 12 anatomic regions was extracted and used for the ROI analysis. For each subject, the T1-weighted MR image volume was used to define the binary ROI with the methods described by Riddle et al. (25). Briefly, the T1-weighted MR image volume of each subject was aligned with a reference brain with a rigid transform ( 3 translation vectors and 3 rotation angles), then the deformation field between the reference brain and the globally aligned T1-weighted MR image volume was calculated with a nonrigid demons registration algorithm $(26,27)$. Binary masks for 12 regions (left and right frontal lobes, cingulate cortex, occipital lobe, left and right parietal lobes, left and right temporal lobes, sublobar region [corpus callosum, caudate, putamen, globus pallidus, thalamus], cerebellum, midbrain, and pons) from the reference brain were warped to the ADNI image volume with the nonrigid deformation field. Figure 1 illustrates these regions in 1 subject. Each brain volume was also segmented into cerebral spinal fluid, gray matter, and white matter with fuzzy C-means (28), and a gray matter mask was created for each of the 12 anatomic regions. The longitudinal ${ }^{18} \mathrm{~F}$-FDG PET images were coregistered with the subject's T1-weighted MR image volume using SPM8 (Wellcome Department of Cognitive Neurology). These, together with the subject's specific gray matter for 12 regions, were loaded into Amide software (29). The mean intensity of each region on the PET image was calculated as a vector of length 12 , which we refer to as ${ }^{18} \mathrm{~F}-\mathrm{FDG}$ vector.

\section{Regional ${ }^{18}$ F-FDG Time Correlation (rFTC)}

For each subject, the Pearson correlation coefficient between the subject's baseline ${ }^{18} \mathrm{~F}-\mathrm{FDG}$ vector and those from the follow-up scans were calculated and displayed as a function of the time $(\mathrm{rFTC}(\mathrm{t}))$. The size of the regions was incorporated as their weighting factor into the 


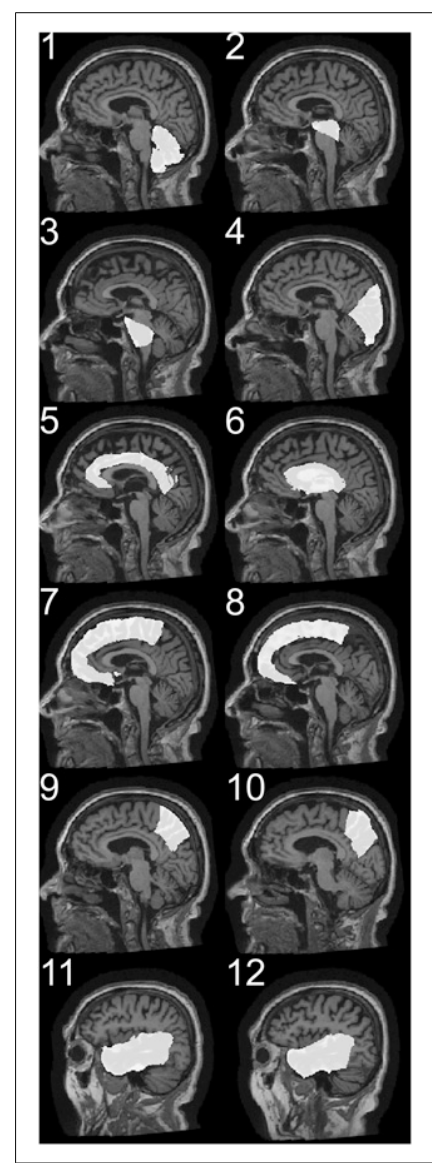

FIGURE 1. Illustration of regional binary masks warped from reference brain to ADNI image volume with nonrigid deformation field. Regions include cerebellum (1); midbrain (2); pons (3); occipital lobe (4); cingulate cortex (5); sublobar region (6) including corpus callosum, caudate, putamen, globus pallidus, thalamus, and right and left frontal lobes $(7,8)$; right and left parietal lobes $(9,10)$; and right and left temporal lobes $(11,12)$. correlation calculation. The correlation coefficient of the initial vector, $\operatorname{rFTC}(\mathrm{t}=0)$, is equal to 1.0 for all subjects. The $r F T C(t)$ value at any subsequent time point at a followup ${ }^{18}$ F-FDG PET scan determines the correlation between the ${ }^{18} \mathrm{~F}-\mathrm{FDG}$ vector at that time point and the baseline vector. Metabolic changes in any of the 12 regions can cause a decline in the $\operatorname{rFTC}(\mathrm{t})$. The decline in rFTC does not strictly indicate a decline in ${ }^{18} \mathrm{~F}$-FDG uptake. The rFTC value represents only similarities between the ${ }^{18} \mathrm{~F}-\mathrm{FDG}$ distributions. Figure 2 shows a comparison between the ${ }^{18} \mathrm{~F}$ FDG vectors at baseline and follow-up in 2 subjects.

\section{Statistical Analyses with Mixed-Effects Model}

The longitudinal changes of rFTC and all 3 cognitive tests and rFTC were analyzed with mixed-effects model using the $\mathrm{R}$ software package (www.rproject.org). We used 2 models for this study. In the first model, the subject's sex, baseline age, time and group specification (MCI $=0$ for normal, MCI $=1$ for mild cognitive impairment), and time by group interaction (time:MCI) were included as fixed effects. This model was performed for $\mathrm{rFTC}$ $(\mathrm{t})$, MMSE(t), ADAS_cog(t), and FAQ( $(t)$ as outcome variables to see how changes in these variables over time differed between MCI and normal subjects. In the second model, the subject's sex, baseline age, time and group specification (APOE), and time by group interaction (time: APOE) were included as fixed effects. The APOE variable was set to 1 for the presence of single or double APOE- $\varepsilon 4$ allele and to 0 otherwise. This model was performed for $r F T C(t), \operatorname{MMSE}(t), \operatorname{ADAS} \_c o g(t)$, and $F A Q(t)$ as outcome variables to see how changes in these variables over time differed between the group with APOE- $\varepsilon 4$ allele and those who do not carry this gene.

\section{Associations Between rFTC(t) and Cognitive Tests}

Two quantitative methods were used to examine the association between $\operatorname{rFTC}(\mathrm{t})$ and cognitive tests in MCI subjects. In the first method (method A), the Spearman rank correlation between the rFTC (t) and each of the cognitive tests was calculated for each individual subject. This method is a simple way to reveal overall associations in decline. In many MCI subjects, the scores of cognitive examinations exhibited temporal fluctuations in their decline. To evaluate whether similar fluctuation patterns also exist in $\operatorname{rFTC}(t)$, we used a partial correlation method (method B). This method measures the degree of association between 2 random variables after the effect of other variables on the 2 variables of interest is removed. Method B was performed by first removing the linear trend of $\operatorname{rFTC}(\mathrm{t}), \operatorname{MMSE}(\mathrm{t})$, $\mathrm{FAQ}(\mathrm{t})$, and ADAS_cog(t) with time, and then calculating the correlations between the fit residuals of $\mathrm{rFTC}$ and each of the 3 cognitive tests. A Wilcoxon rank-sum test was performed on both methods A and B to determine associations in group level.

\section{RESULTS}

\section{rFTC Measures Over Time}

The $\operatorname{rFTC}(\mathrm{t})$ of all subjects is illustrated in Figure 3. The rFTC values of controls remained fairly high over time $(-0.003$ annual change; $95 \%$ confidence interval, $-0.010-0.004)$. In MCI patients, the rFTC declined faster than in controls by an additional annual change of -0.02 ( $95 \%$ confidence interval, -0.030 to -0.010 ). The results of both mixed-effects models are summarized in Table 2. The estimate of the variable produced by the model determines the group difference, whereas the $P$ value indicates the statistical significance of the estimate. Because we are interested in the change over time, baseline values of ADAS_cog, MMSE, and FAQ were not included in this model. Although the baseline values of cognitive scores are different between MCI and normal subjects (Table 1), the baseline rFTC is 1.0 for all subjects, because follow-up scans are compared with the initial scan.

\section{Correlation Between rFTC and Cognitive Status}

The relationship between $\operatorname{rFTC}(\mathrm{t})$ and the time course of cognitive status as measured using the ADAS_cog, MMSE, and FAQ was evaluated with 2 correlation methods. The Wilcoxon ranksum results of the Spearman rank correlation between $\operatorname{rFTC}(\mathrm{t})$ and the longitudinal cognitive test scores in MCI subjects and the

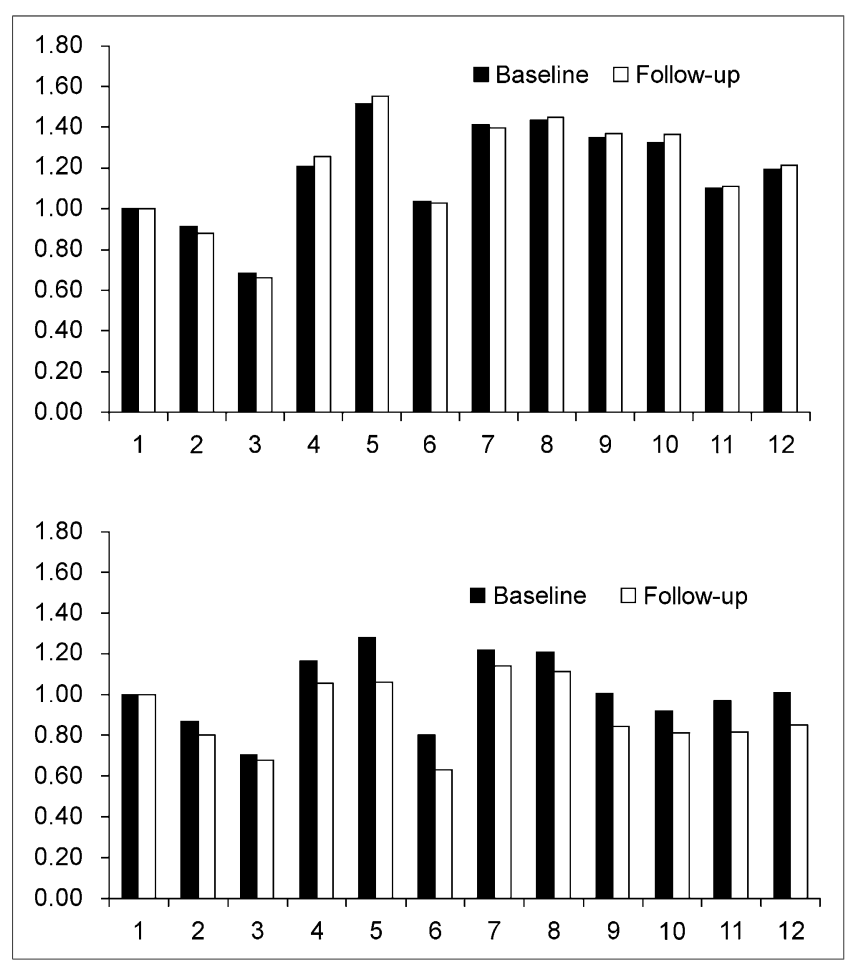

FIGURE 2. Comparison between ${ }^{18} \mathrm{~F}-\mathrm{FDG}$ vectors at baseline and follow-up in 2 subjects. Lower figure shows subject with low correlation, and upper figure shows subject with high correlation. 


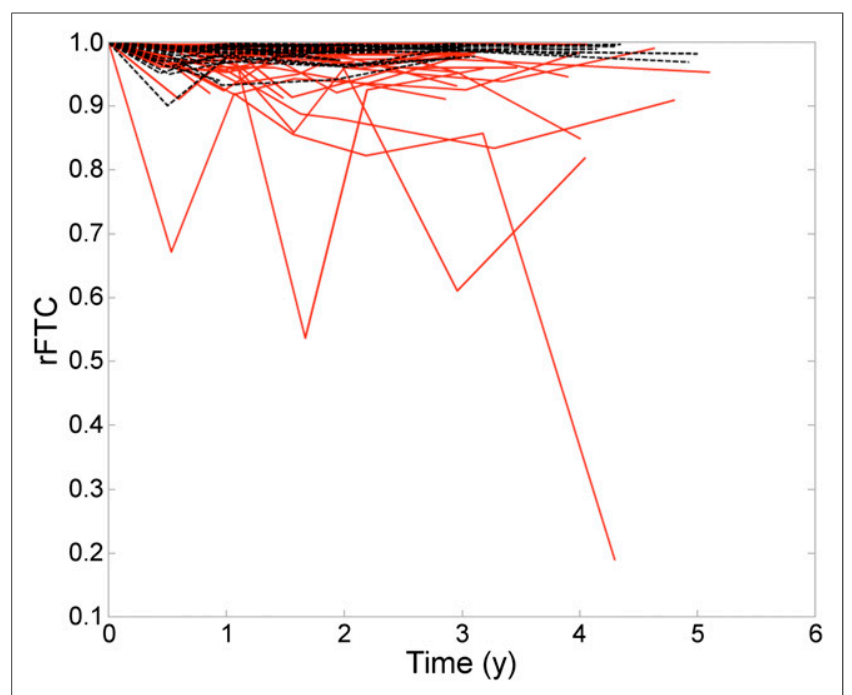

FIGURE 3. Comparison between normal (black dashed line) and $\mathrm{MCl}$ (red solid line) subjects with respect to their $\mathrm{rFTC}(\mathrm{t})$.

results of the partial correlations (method B) are summarized in Table 3. Although the median and $P$ values in method A represent overall similarities over time and their corresponding statistical significance, the median and $P$ values of method B show partial correlations, thus reflecting similarities in fluctuation patterns. The similarity between $\mathrm{rFTC}(\mathrm{t})$ and longitudinal changes in cognitive status within an individual subject is exemplified by Figures 4 and 5. Figure 4 illustrates the association between temporal changes of rFTC(t) and the cognitive tests in an MCI subject with large cognitive decline. Figure 5 illustrates the association between temporal changes of $\mathrm{rFTC}(\mathrm{t})$ and the cognitive tests in a MCI subject with moderate cognitive decline.

\section{DISCUSSION}

Although previous methods, such as PALZ (20) or MetaROI (19), included brain regions that are typically affected in AD, we

TABLE 2

Outcome Results of 2 Mixed-Effects Models

\begin{tabular}{lccc}
\hline \multicolumn{1}{c}{ Model } & Fixed effect & Estimate & $P$ \\
\hline 1 & & & \\
ADAS_cog & time:MCl & 1.6 & 0.0001 \\
rFTC & time:MCI & -0.02 & 0.0001 \\
MMSE & time: $\mathrm{MCl}$ & -0.82 & 0.0002 \\
FAQ & time:MCl & 2.5 & 0.0001 \\
2 & & & \\
ADAS_cog & time:APOE & 1.14 & 0.001 \\
rFTC & time:APOE & -0.014 & 0.008 \\
MMSE & time:APOE & -0.44 & 0.007 \\
FAQ & time:APOE & 1.32 & 0.0001 \\
\end{tabular}

Both models were performed for $r F T C(t)$, MMSE(t), ADAS-cog $(t)$, and $F A Q(t)$ as outcome variables. With the first model (time: $\mathrm{MCl}$ ), we see how changes in these variables over time differed between $\mathrm{MCl}$ and normal subjects. Second model (time:APOE) shows how changes in these variables over time differed between the group with positive APOE- $\varepsilon 4$ and control group. selected 12 regions that cover the entire brain to perform a correlation analysis. In general, conventional ${ }^{18} \mathrm{~F}-\mathrm{FDG}$ analyses rely on the location and quantification of ${ }^{18} \mathrm{~F}$-FDG uptake measurements, whereas our method highlights similarities between the subject's serial scans using a parameter that we term the rFTC. The rFTC decreases significantly faster in subjects from the risk groups (MCI, APOE- $\varepsilon 4$ ) than in controls (Table 2). Although differences in PET camera, data acquisition, test-retest variability of ${ }^{18} \mathrm{~F}-\mathrm{FDG}$ PET, and increases of ${ }^{18}$ F-FDG uptake could also lead to decreased correlation, we showed that the rFTC parameter is fairly stable in controls - that is, control subjects maintain a higher correlation between their baseline and follow-up ${ }^{18} \mathrm{~F}-\mathrm{FDG}$ vectors. Also, we limited our subject selection to those whose serial ${ }^{18} \mathrm{~F}$ FDG PET scans were obtained with the same scanner, acquisition protocol, and image reconstruction method at baseline and each follow-up to reduce variations in data acquisition.

We saw that in MCI subjects, greater rFTC decline was associated with a greater decline in ADAS_cog, FAQ, and MMSE (Table 3). In other words, MCI subjects with less cognitive decline also had less rFTC decline. Our method allows a direct comparison between the metabolic state and the cognitive score using a simple and individualized approach, as represented by $2 \mathrm{MCI}$ subjects in Figures 4 and 5. Empiric examples emphasize the potential of ${ }^{18} \mathrm{~F}-\mathrm{FDG}$ PET to assess AD treatment efficacies (30). Given the current interest in developing treatments that can slow or halt the AD progression, rFTC outcomes offer the potential to highlight simultaneous metabolic and cognitive changes for tracking response to therapy. This method can be easily combined with validated ${ }^{18} \mathrm{~F}$-FDG PET clinical assessments using PET data acquired by standard clinical protocols.

The rFTC method does not directly capture the size, location, and extent of the regions with metabolic changes. We applied large anatomic ROIs to assess regional effects over time. A smallerregion approach may offer greater regional specificity in characterizing the decline of rFTC. However, warping masks from a reference brain to a subject's brain with the nonrigid demons registration algorithm works well for large structures such as the cerebellum but not for small internal structures such as the caudate or the hippocampus because of differences between brains (27). One of our future goals is to overcome this challenge by evaluating a variety of algorithms that can accurately warp or define smaller structures in a consistent way for all the subjects. A voxel-based correlation analysis could eliminate the issues related to the regional segmentation. However, a voxel-based approach is more susceptible to the noise variations in ${ }^{18} \mathrm{~F}$-FDG image voxel

TABLE 3

Correlation Between rFTC and Cognitive Tests

\begin{tabular}{lccccc}
\hline & \multicolumn{3}{c}{ Method $A$} & & \multicolumn{2}{c}{ Method B } \\
\cline { 2 - 3 } \cline { 5 - 6 } Variable & Median & $P$ & & Median & $P$ \\
\hline ADAS-cog/rFTC & 0.5 & 0.011 & & 0.81 & 0.0005 \\
MMSE/rFTC & 0.5 & 0.0039 & & 0.64 & 0.00004 \\
FAQ/rFTC & 0.6 & 0.0016 & & 0.4 & 0.0002
\end{tabular}

Wilcoxon rank-sum tests of Spearman rank correlation between rFTC and each of 3 cognitive tests (method A) and correlation between residuals of linear regressions on $\mathrm{rFTC}(\mathrm{t})$ and 3 cognitive tests (method B). 


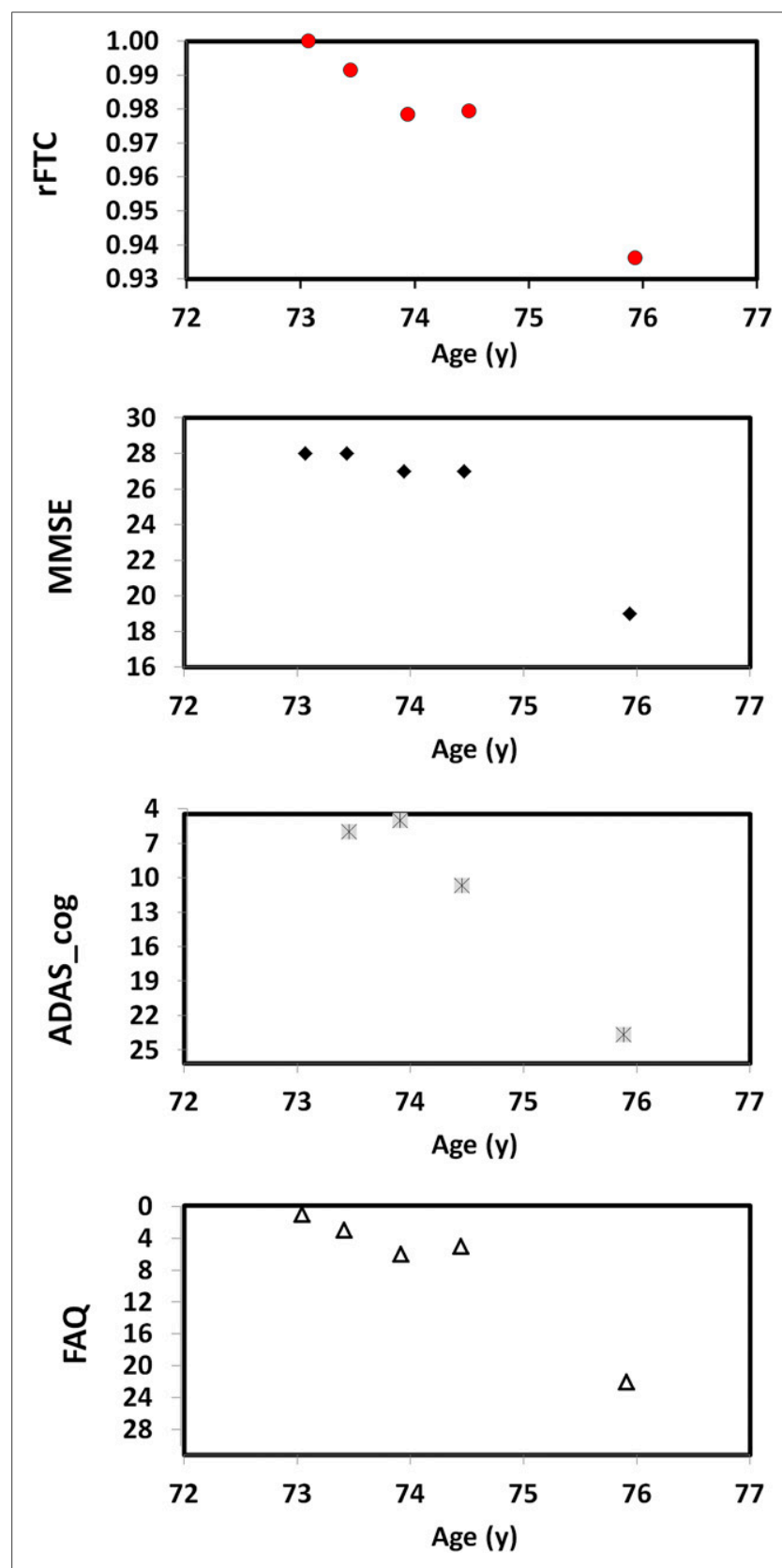

FIGURE 4. Changes in rFTC, MMSE, FAQ, and ADAS_cog values with age in $\mathrm{MCl}$ subject who has large decline in cognitive scores.

values, which can inhibit the observation of metabolic variations. Therefore, one of our optimization objectives is to find a trade-off in the spatial scale between voxels and larger ROIs to provide both sufficient signal-to-noise ratio and spatial specificity in detecting rFTC decline. We are also interested in performing a correlation analysis on regions based on functional connectivity criteria, instead of the conventional anatomic approach. This analysis could be achieved by incorporating the default-mode activity regions (31) at individual levels from a subject's functional connectivity MR imaging analyses to address which areas within this network contribute to the highest rFTC decline over time or the greatest association with cognitive ratings.
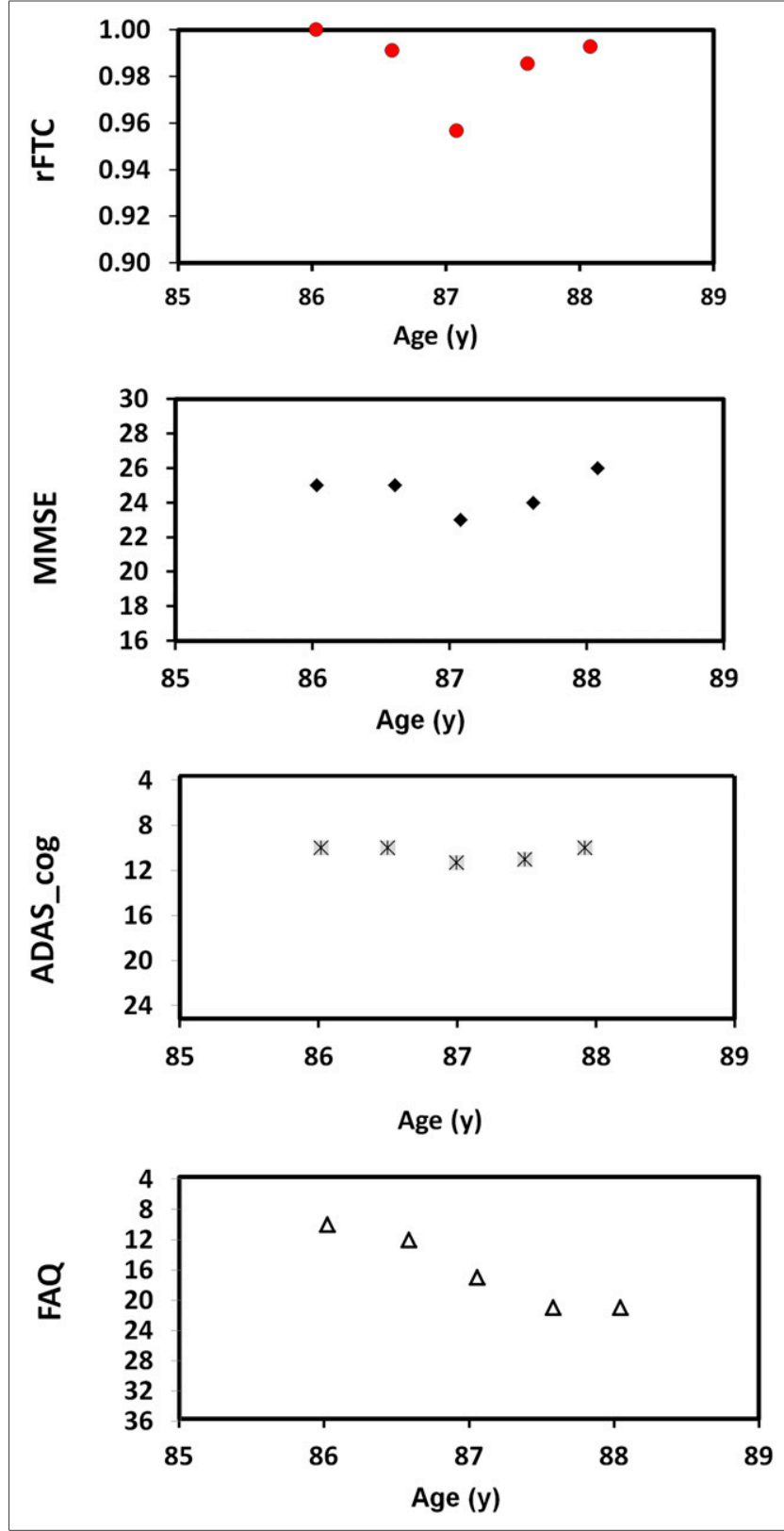

FIGURE 5. Changes in rFTC, MMSE, FAQ, and ADAS_cog values with age in $\mathrm{MCl}$ subject who has moderate decline in cognitive scores.

\section{CONCLUSION}

Longitudinal changes in rFTC detect subtle metabolic changes associated with variations in cognition. This analytic tool may be useful for monitoring cognitive decline in individual subjects.

\section{DISCLOSURE}

The costs of publication of this article were defrayed in part by the payment of page charges. Therefore, and solely to indicate this fact, this article is hereby marked "advertisement" in accordance with 18 USC section 1734. Data collection and sharing for this project was funded by the Alzheimer's Disease Neuroimaging Initiative (ADNI) (National Institutes of Health grant U01 AG024904). 
ADNI is funded by the National Institute on Aging, the National Institute of Biomedical Imaging and Bioengineering, and through generous contributions from the following: Abbott; Alzheimer's Association; Alzheimer's Drug Discovery Foundation; Amorfix Life Sciences Ltd.; AstraZeneca; Bayer HealthCare; BioClinica, Inc.; Biogen Idec Inc.; Bristol-Myers Squibb Company; Eisai Inc.; Elan Pharmaceuticals Inc.; Eli Lilly and Company; F. Hoffmann-La Roche Ltd and its affiliated company Genentech, Inc.; GE Healthcare; Innogenetics, N.V.; IXICO Ltd.; Janssen Alzheimer Immunotherapy Research \& Development, LLC.; Johnson \& Johnson Pharmaceutical Research \& Development LLC.; Medpace, Inc.; Merck \& Co., Inc.; Meso Scale Diagnostics, LLC.; Novartis Pharmaceuticals Corporation; Pfizer Inc.; Servier; Synarc Inc.; and Takeda Pharmaceutical Company. The Canadian Institutes of Health Research is providing funds to support ADNI clinical sites in Canada. Private sector contributions are facilitated by the Foundation for the National Institutes of Health (www.fnih.org). The grantee organization is the Northern California Institute for Research and Education, and the study is coordinated by the Alzheimer's Disease Cooperative Study at the University of California, San Diego. ADNI data are disseminated by the Laboratory for Neuro Imaging at the University of California, Los Angeles. This research was also supported by NIH grants P30 AG010129 and K01 AG030514 and by NIH/NIBIB K99 EB 009106, NIH R01 CA109106, and NIH R01 EB000461. No other potential conflict of interest relevant to this article was reported.

\section{ACKNOWLEDGMENTS}

We thank Dominique Delbeke, Todd Peterson, Noor Tantawy, Angela Jefferson, and John Gore from Vanderbilt University for supportive discussions and Dr. Benoit M. Dawant and Rui Li for providing the rigid and demons registration software. Data used in preparation of this article were obtained from the ADNI database (adni.loni.ucla.edu). As such, the investigators within the ADNI contributed to the design and implementation of ADNI and/or provided data but did not participate in analysis or writing of this report. A complete listing of ADNI investigators can be found at: http://adni.loni.ucla.edu/wp- content/uploads/how_to_apply/ADNI_ Acknowledgment_List.pdf.

\section{REFERENCES}

1. Flicker C, Ferris SH, Reisberg B. Mild cognitive impairment in the elderly: predictors of dementia. Neurology. 1991;41:1006-1009.

2. Petersen RC, Smith GE, Waring SC, Ivnik RJ, Tangalos EG, Kokmen E. Mild cognitive impairment: clinical characterization and outcome. Arch Neurol. 1999; 56:303-308.

3. Morris JC, Storandt M, Miller JP, et al. Mild cognitive impairment represents early-stage Alzheimer disease. Arch Neurol. 2001;58:397-405.

4. Visser PJ, Salmon E. Role of cognitive testing in disease modifying AD trials. J Nutr Health Aging. 2006;10:131-132.

5. Komarova NL, Thalhauser CJ. High degree of heterogeneity in Alzheimer's disease progression patterns. PLoS Computional Biol. 2011;7:e1002251.

6. Jack CR Jr, Slomkowski M, Gracon S, et al. MR imaging as a biomarker of disease progression in therapeutic trial of milameline for AD. Neurology. 2003;60:253-260.

7. Sokoloff L. Relationship among local functional activity, energy metabolism, and blood flow in the central nervous systems. Fed Proc. 1981;40:2311-2316.
8. Minoshima S, Giordani B, Berent S, Frey KA, Foster NL, Kuhl DE. Metabolic reduction in the posterior cingulate cortex in early Alzheimer's disease. Ann Neurol. 1997;42:85-94.

9. Friedland RP, Budinger TF, Ganz E, et al. Regional cerebral metabolic alterations in dementia of the Alzheimer type: positron emission tomography with $\left[{ }^{18}\right.$ F]fluorodeoxyglucose. J Comput Assist Tomogr. 1983;7:590-598.

10. Salmon E, Collette F, Degueldre C, Lemaire C, Franck G. Voxel-based analysis of confounding effects of age and dementia severity on cerebral metabolism in Alzheimer's disease. Hum Brain Mapp. 2000;10:39-48.

11. Silverman DH, Small GW, Chang CY, et al. Positron emission tomography in evaluation of dementia: regional brain metabolism and long-term clinical outcome. JAMA. 2001;286:2120-2127.

12. Reiman EM, Langbaum JB, Tariot PN. Alzheimer's Prevention Initiative: a proposal to evaluate presymptomatic treatments as quickly as possible. Biomark Med. 2010;4:3-14.

13. Jagust WJ, Friedland RP, Budinger TF, Koss E, Ober B. Longitudinal studies of regional cerebral metabolism in Alzheimer's disease. Neurology. 1988;38:909-912.

14. McGeer EG, Peppard RP, McGeer PL, et al. ${ }^{18}$ Fluorodeoxyglucose positron emission tomography studies in presumed Alzheimer cases, including 13 serial scans. Can J Neurol Sci. 1990;17:1-11.

15. Alexander GE, Chen K, Pietrini P, Rapoport SI, Reiman EM. Longitudinal PET evaluation of cerebral metabolic decline in dementia: a potential outcome measure in Alzheimer's Disease Treatment Studies. Am J Psychiatry. 2002;159:738-745.

16. Fouquet M, Desgranges B, Landeau B, et al. Longitudinal brain metabolic changes from amnestic mild cognitive impairment to Alzheimer's disease. Brain. 2009;132:2058-2067.

17. Chen K, Langbaum JBS, Fleisher AS, et al. ADNI: Twelve-month metabolic declines in probably Alzheimer's disease and amnestic mild cognitive impairment assessed using an empirically pre-defined statistical region of interest: findings from the Alzheimer's Disease Neuroimaging Initiative. Neuroimage. 2010;51:654-664.

18. Haxby JV, Grady CL, Koss E, et al. Longitudinal study of cerebral metabolic asymmetries and associated neuropsychological patterns in early dementia of the Alzheimer type. Arch Neurol. 1990;47:753-760.

19. Landau SM, Harvey D, Madison CM, et al. Associations between cognitive, functional, and FDG-PET measures of decline in AD and MCI. Neurobiol Aging. 2011;32:1207-1218.

20. Herholz K, Westwood S, Haense C, Dunn G. Evaluation of a calibrated ${ }^{18}$ F-FDG PET score as a biomarker for progression in Alzheimer disease and mild cognitive impairment. J Nucl Med. 2011;52:1218-1226.

21. Munro CA, Workman CI, Kramer E, et al. Serotonin modulation of cerebral glucose metabolism: sex and age effects. Synapse. 2012;66:955-964.

22. Rosen WG, Mohs RC. Davis KL. A new rating scale for Alzheimer's disease. Am J Psychiatry. 1984;141:1356-1364.

23. Folstein MF, Folstein SE, McHugh PR. Mini-mental state: a practical method for grading the cognitive state of patients for the clinician. J Psychiatr Res. 1975;12: 189-198.

24. Pfeffer RI, Kurosaki TT, Harrah CH Jr, Chance JM. Measurement of functional activities in older adults in the community. J Gerontol. 1982;37:323-329.

25. Riddle WR, DonLevy SC, Wushensky CA, Dawant BM, Fitzpatrick JM, Price RR. Quantifying cerebral changes in adolescence with MR imaging and deformation based morphometry. J Magn Reson Imaging. 2008;28:320-326.

26. Thirion JP. Image matching as a diffusion process: an analogy with Maxwell's demons. Med Image Anal. 1998;2:243-260.

27. Dawant BM, Hartmann SL, Thirion JP, Maes F, Vandermeulen D, Demaerel P. Automatic 3-D segmentation of internal structures of the head in MR images using a combination of similarity and free-form transformations: part I, methodology and validation on normal subjects. IEEE Trans Med Imaging. 1999;18:909-916.

28. Pham DL, Prince JL. Adaptive fuzzy segmentation of magnetic resonance images. IEEE Trans Med Imaging. 1999;18:737-752.

29. Loening AM, Gambhir SS. AMIDE: A free software tool for multimodality medical image analysis. Mol Imaging. 2003;2:131-137.

30. Rogers SL, Farlow MR, Doody RS, Mohs R, Friedhoff LT. A 24-week, doubleblind, placebo-controlled trial of donepezil in patients with Alzheimer's disease. Donepezil Study Group. Neurology. 1998;50:136-145.

31. Buckner RL, Andrews-Hanna JR, Schacter DL. The brain's default network: anatomy, function, and relevance to disease. Ann N Y Acad Sci. 2008;1124:1-38. 